\title{
Crypton transposons: identification of new diverse families and ancient domestication events
}

Kenji K Kojima and Jerzy Jurka*

\begin{abstract}
Background: "Domestication" of transposable elements (TEs) led to evolutionary breakthroughs such as the origin of telomerase and the vertebrate adaptive immune system. These breakthroughs were accomplished by the adaptation of molecular functions essential for TEs, such as reverse transcription, DNA cutting and ligation or DNA binding. Cryptons represent a unique class of DNA transposons using tyrosine recombinase (YR) to cut and rejoin the recombining DNA molecules. Cryptons were originally identified in fungi and later in the sea anemone, sea urchin and insects.
\end{abstract}

Results: Herein we report new Cryptons from animals, fungi, oomycetes and diatom, as well as widely conserved genes derived from ancient Crypton domestication events. Phylogenetic analysis based on the YR sequences supports four deep divisions of Crypton elements. We found that the domain of unknown function 3504 (DUF3504) in eukaryotes is derived from Crypton YR. DUF3504 is similar to YR but lacks most of the residues of the catalytic tetrad (R-H-R-Y). Genes containing the DUF3504 domain are potassium channel tetramerization domain containing 1 (KCTD1), KIAA1958, zinc finger MYM type 2 (ZMYM2), ZMYM3, ZMYM4, glutamine-rich protein 1 (QRICH1) and "without children" (WOC). The DUF3504 genes are highly conserved and are found in almost all jawed vertebrates. The sequence, domain structure, intron positions and synteny blocks support the view that ZMYM2, ZMYM3, ZMYM4, and possibly QRICH1, were derived from WOC through two rounds of genome duplication in early vertebrate evolution. WOC is observed widely among bilaterians. There could be four independent events of Crypton domestication, and one of them, generating WOC/ZMYM, predated the birth of bilaterian animals. This is the third-oldest domestication event known to date, following the domestication generating telomerase reverse transcriptase (TERT) and Prp8. Many Crypton-derived genes are transcriptional regulators with additional DNAbinding domains, and the acquisition of the DUF3504 domain could have added new regulatory pathways via protein-DNA or protein-protein interactions.

Conclusions: Cryptons have contributed to animal evolution through domestication of their YR sequences. The DUF3504 domains are domesticated YRs of animal Crypton elements.

Keywords: tyrosine recombinase, Crypton, domestication, transposon, DUF3504

\section{Background}

The structural and mechanistic variety of transposable elements (TEs) is well-documented [1]. They encode proteins that include diverse functional domains involved in catalysis or interaction with DNA, RNA and other proteins. Because of this diverse repertoire, TEs can supply functional modules to generate new genes. "Molecular domestication" of transposable elements [2] led to evolutionary

\footnotetext{
* Correspondence: jurka@girinst.org

Genetic Information Research Institute, 1925 Landings Drive, Mountain View, CA 94043, USA
}

milestones such as the origin of telomerase and the vertebrate adaptive immune system. Telomerase reverse transcriptase (TERT) provides a solution for end replication problems accompanying linear chromosome replication and was derived from a reverse transcriptase (RT) related to Penelope-like elements in the very early stage of eukaryote evolution $[3,4]$. $\mathrm{V}(\mathrm{D}) \mathrm{J}$ recombination is a mechanism used in jawed vertebrates to generate a variety of immunoglobulins and $\mathrm{T}$-cell receptors. It is catalyzed by the recombination activating gene 1 (RAG1) derived from a transposase encoded by the Transib family of DNA transposons [5]. Different kinds of transposon proteins were

\section{Biomed Central}


domesticated, including transposase, integrase, RT, envelope and gag proteins [6]. Herein we report in-depth studies of another type of transposon enzyme, tyrosine recombinase (YR), which was repeatedly domesticated in the history of animals.

To date four types of enzymes are known to catalyze DNA integration of eukaryotic transposons: DDE-transposase, YR, rolling-circle replication initiator and the combination of RT and endonuclease (EN) [7]. DDEtransposase is the most abundant gene in nature [8] and is carried by many DNA transposon superfamilies, selfsynthesizing transposons (Polinton), as well as long terminal repeat (LTR) retrotransposons (Gypsy, Copia, BEL and endogenous retroviruses) [1,9-11]. They share three conserved amino acids (DDD or DDE) at their catalytic sites, which are separated by amino acid sequences of varying length. Some domesticated DDE-transposases became DNA-binding proteins, such as CENP-B in mammals and Daysleeper in Arabidopsis thaliana [12,13]. Non-LTR retrotransposons and Penelope-like elements use a combination of RT and EN in their transposition [14-17]. Helitron is the only group of eukaryotic transposons encoding rolling-circle replication initiator [9].

YR genes are ubiquitous in prokaryotes but rare in eukaryotes $[18,19]$. All YRs found in eukaryotes are encoded by mobile elements: yeast 2 -micron circle plasmids [20], ciliate Euplotes crassus transposons (Tec1, $T e c 2$ and Tec3) [21,22], three groups of retrotransposons (DIRS/Pat, Ngaro and VIPER) [23-25], and Cryptons [19]. The YR encoded by the yeast 2 -micron plasmid, known as "flippase" (FLP), is widely used for site-specific recombination in the FLP-FRT system [26]. Tec1 and Tec2 transposons encode a DDE-transposase in addition to YR, and therefore the YR domains in these transposons are probably involved in resolving transposition intermediates. To date the only YR-encoding transposons found in the vertebrate genomes are DIRS and Ngaro retrotransposons. Cryptons were originally found in a basidiomycete Cryptococcus neoformans and several pathogenic fungi. Their boundaries are difficult to characterize because they have neither terminal inverted repeats (TIRs) nor long direct repeats. Instead they have short direct repeats at both termini. These 4- or 6-bp direct repeats are considered substrates for recombination. By analogy to prokaryotic YR-encoding transposons, Goodwin et al. [19] proposed that Cryptons are excised from the host genome as an extrachromosomal circular DNA and integrated at a different locus in the genome. YR typically recognizes recombination sites consisting of two inverted repeats that are 11 to $13 \mathrm{bp}$ long and separated by a segment 6 to 8 bp long [27]. Recently, transposons encoding only a YR have been found in sea urchin, insects and cnidarians and classified as Cryptons [28,29]. YR contains four catalytically important residues (R-H-R-
$\mathrm{Y})$, but their overall sequence identity is very low among different genes and transposons [18,19]. The conserved tyrosine residue directly binds to DNA in the recombination reaction. In this paper, we report Cryptons from various species, including medaka fish, and six human genes originated from ancient domestication events of Crypton YRs.

\section{Results}

The diversity of Crypton elements in terms of their sequence and domain structure

We identified 94 Crypton elements from 24 species representing animals, fungi and stramenopiles that include oomycetes and diatom (Figure 1, Table 1 and Additional file 1). Phylogenetic clustering of Cryptons on the basis of their YR domain sequences revealed four groups reflecting the systematics of their hosts (Figure 2, open circles), but two of them were not strongly supported phylogenetically because of the low bootstrap values. Herein we designate them as CryptonF, CryptonS, CryptonA and CryptonI to indicate their corresponding hosts: fungi, stramenopiles, animals and insects. CryptonA and CryptonI are structurally similar; however, CryptonF, CryptonS and CryptonA/CryptonI have distinct protein domain structures (see Figure 1 and detailed description in the next three sections). Because of the low resolution of the phylogenetic tree, we could not determine whether there is any relationship between these four Crypton groups and to other YR-encoding elements, and we cannot rule out the possibility that they have originated independently.

\section{CryptonF elements from fungi and oomycetes, and CryptonF-derived genes}

We identified CryptonF elements in nine species of fungi and four species of oomycetes (Table 1 and Additional file 1). These elements encode a protein that includes YR and GCR1_C DNA-binding domains (Figure 1). Most of the fungal Cryptons and the five oomycete Cryptons are associated with 6-bp terminal direct repeats, which are likely substrates for Crypton integration (Additional file 1). In Fusarium oxysporum, Crypton is fused with a Marinertype DNA transposon and this composite transposon is hearafter named MarCry-1_FO (Figure 1). The analysis of four MarCry-1_FO copies with more than 97\% identity to each other revealed the presence of 16-bp TIRs and target site duplications (TSDs) of the TA dinucleotide, indicating that their Mariner-type DDE-transposase is responsible for transposition. CryptonF-2_PS from Phytophthora sojae and related elements encode a $\mathrm{C} 48$ peptidase (Ulp1 protease) in addition to a YR (Figure 1). The oomycete CryptonF elements are nested in fungal CryptonF elements in the phylogenetic tree (Figure 2), indicating a horizontal transfer between fungi and oomycetes. 


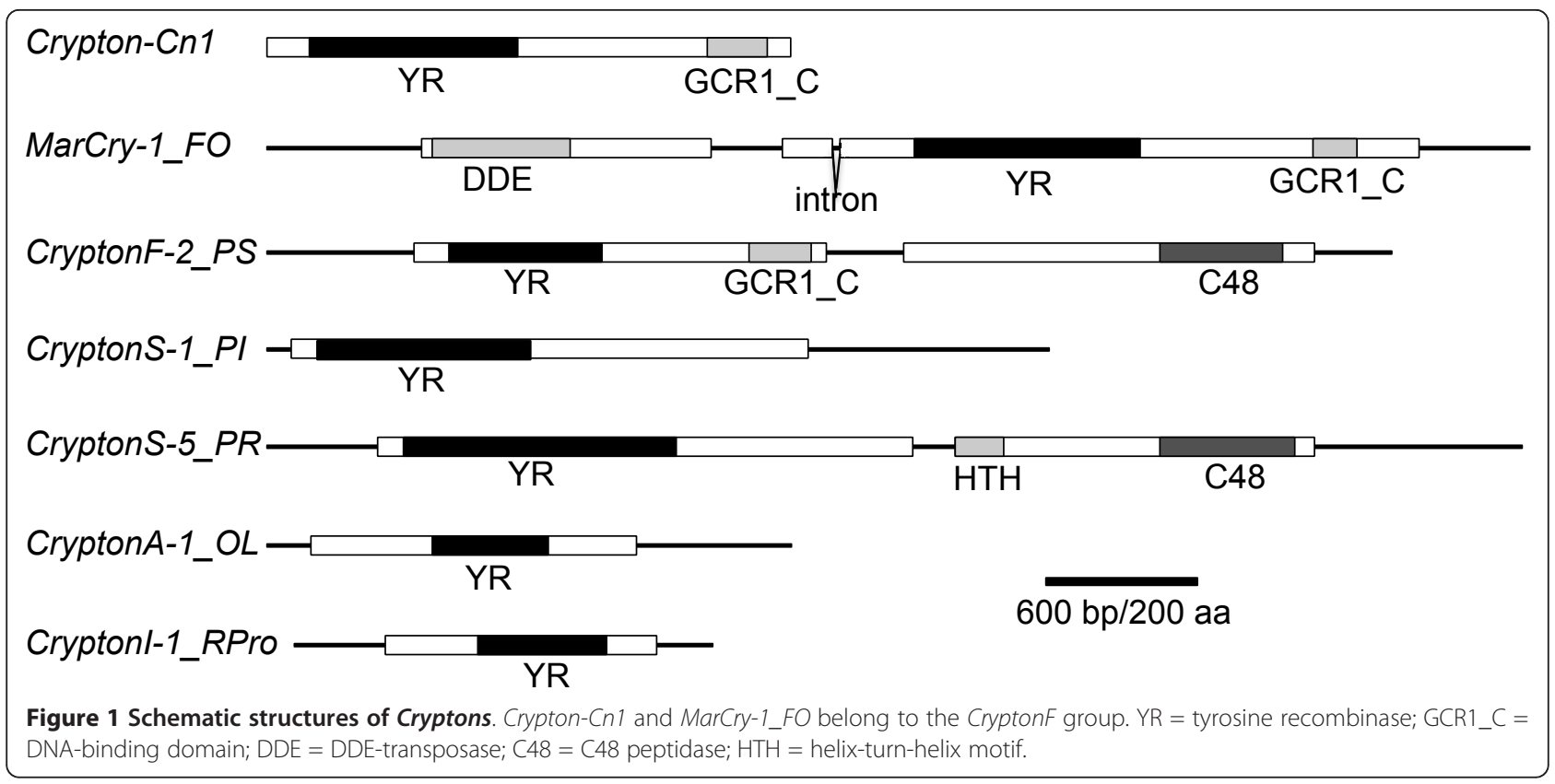

Four genes from Saccharomyces cerevisiae were derived from CryptonF elements (Figure 3 and Additional files 2 and 3). It was previously reported that the GCR1_C protein domain encoded by Gor $1, M s n 1$ and Hot 1 genes is similar to the C-terminal part of fungal Cryptons [19]. In addition to these three genes, we found that $\mathrm{Cbf} 2 / \mathrm{Ndc10}$ contains a C-terminal domain similar to CryptonF proteins. The central portions of Cbf2 and Gcr1 are similar to CryptonF YR domains, but the catalytic site is not preserved (data not shown). Vanderwaltozyma polyspora carries two paralogous genes of Gcr1 and Msn1. Candida tropicalis and related species (Candida albicans, Pichia stipitis and Pichia guilliermondii) harbor another gene derived from a CryptonF element, represented by
XP_002548716 in C. tropicalis. It is designated herein as Crypton-derived gene 1 (Cdg1) (Figure 3 ). The only domain shared by CryptonF elements and all Cryptonderived genes is the GCR1_C domain. The phylogenetic analysis of GCR1_C domains (Figure 3C) indicates that Hot 1 and $M s n 1$ are paralogous and that the gene related to Hot1/Msn1 in C. tropicalis represents an outgroup of both genes. Therefore, it is likely that four domestication events (for Hot1/Msn1, Gcr1, Cbf2 and Cdg1) occurred in this group.

We could not find any Crypton insertions in the subphylum Saccharomycotina (including S. cerevisiae, C. tropicalis and related species). The distribution of Crypton-derived genes indicates that Crypton was active

Table 1 Distribution of Crypton elements

\begin{tabular}{|c|c|c|}
\hline Classification & $\begin{array}{l}\text { Phylum or } \\
\text { Class }\end{array}$ & Species $^{a}$ \\
\hline \multirow[t]{3}{*}{ Fungi } & Basidiomycota & Cryptococcus neoformans [19] \\
\hline & Ascomycota & $\begin{array}{l}\text { Coccidioides posadasii [19], Histoplasma capsulatum [19], Chaetomium globosum, Fusarium oxysporum, Ajellomyces } \\
\text { capsulatus, Coccidioides immitis, Microsporan canis, Talaromyces stipitatus, Neosartorya fischeri }\end{array}$ \\
\hline & Zygomycota & Rhizopus oryzae \\
\hline \multirow[t]{6}{*}{ Animals } & Chordata & Oryzias latipes \\
\hline & Echinodermata & Strongylocentrotus purpuratus [28] \\
\hline & Hemichordata & Saccoglossus kowalevskii \\
\hline & Mollusca & Lottia gigantea \\
\hline & Arthropoda & Nasonia vitripennis [29], Tribolium castaneum [29], Rhodnius prolixus, Aedes aegypti, Culex quinquefasciatus \\
\hline & Cnidaria & Nematostella vectensis [28] \\
\hline \multirow[t]{2}{*}{ Stramenopiles } & Oomycetes & $\begin{array}{l}\text { Phytophthora infestans, Phytophthora sojae, Phytophthora ramorum, Pythium ultimum, Saprolegnia parasitica, } \\
\text { Hyaloperonospora arabidopsidis, Albugo laibachii }\end{array}$ \\
\hline & Diatoms & Phaeodactylum tricornutum \\
\hline
\end{tabular}

${ }^{a}$ Crypton elements from species without references are found in this study. 


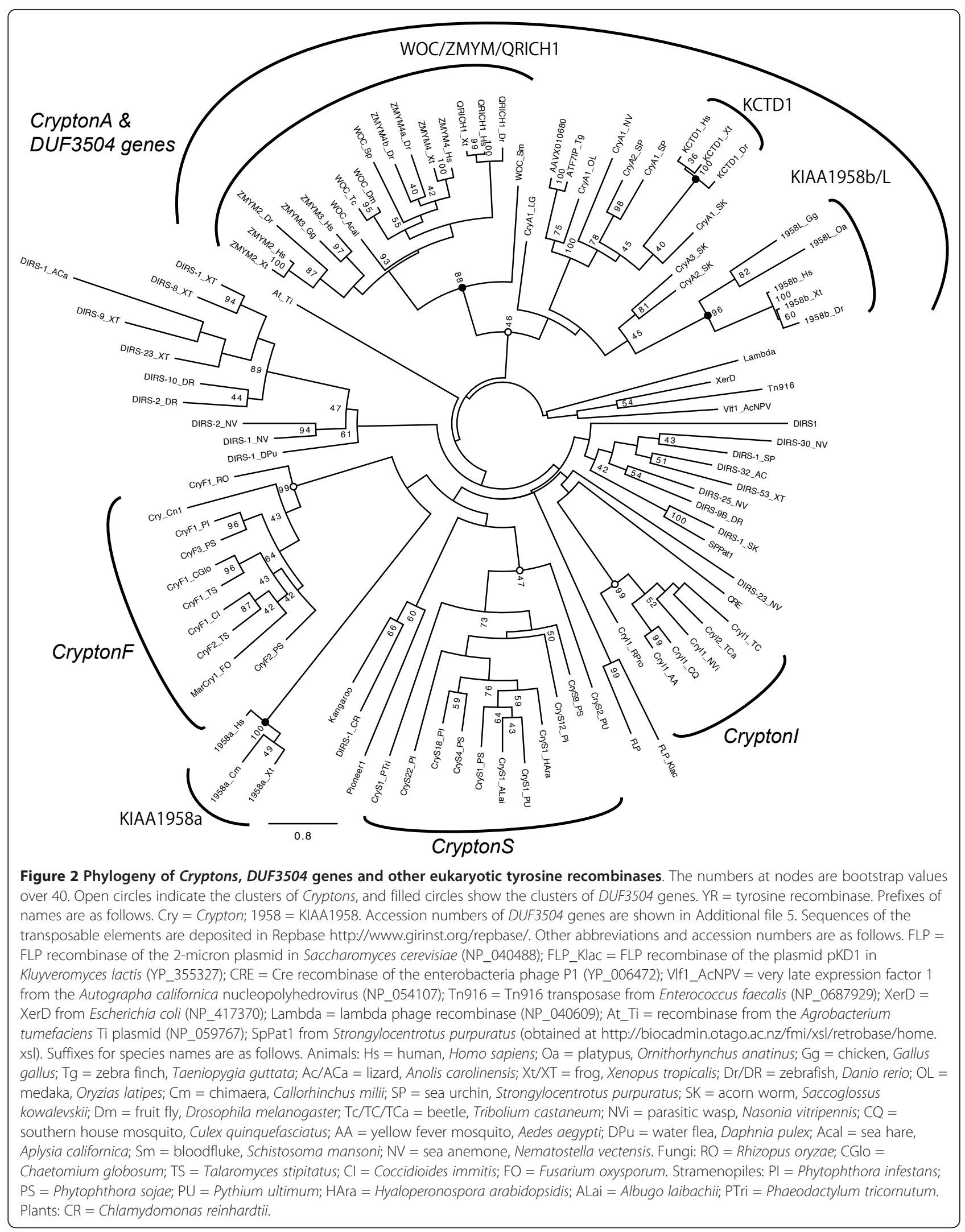




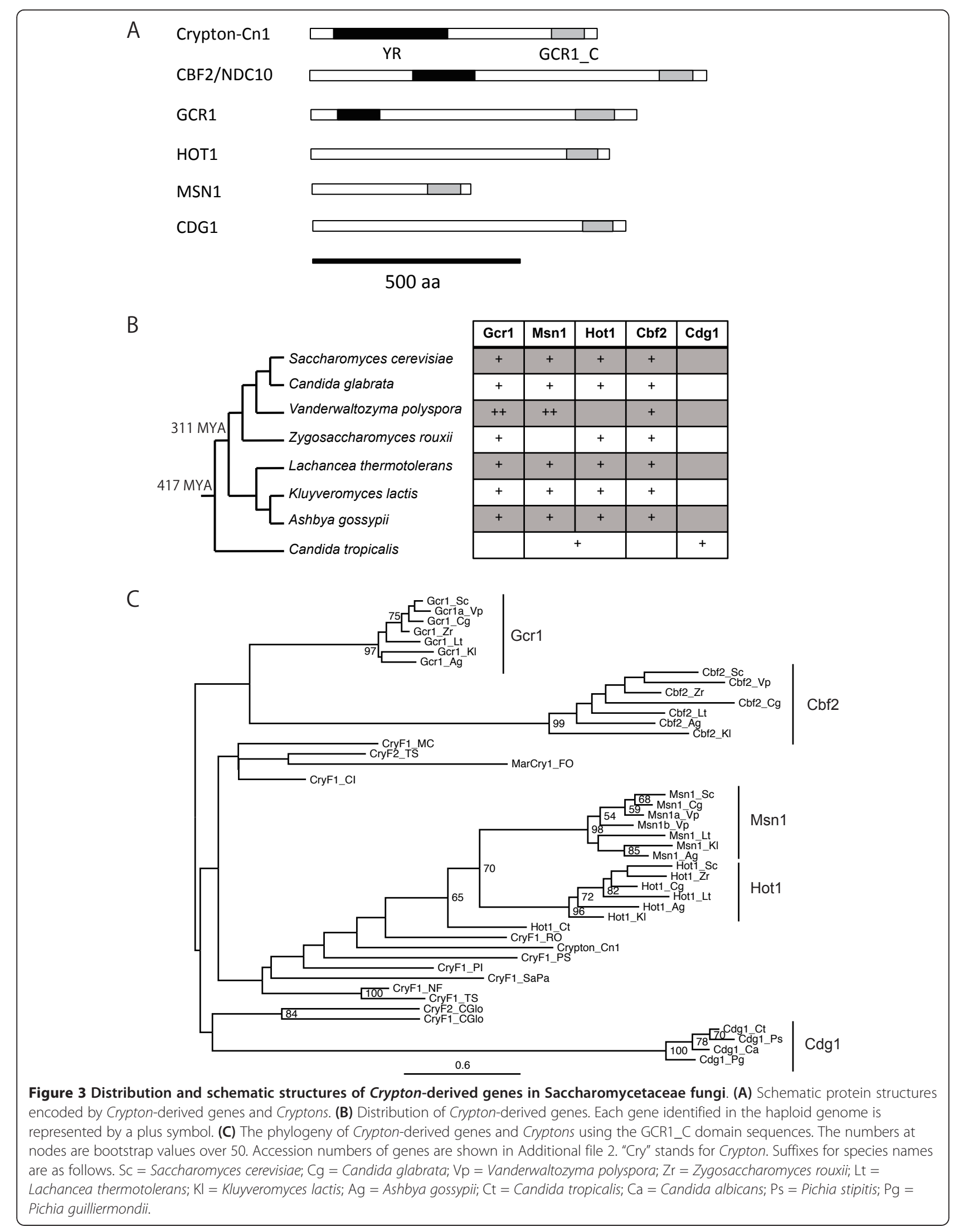


in the past and that the DNA-binding domain GCR1_C was most likely derived from Cryptons.

\section{Cryptons, a new group of Cryptons from oomycetes and diatom}

We found CryptonS elements in seven oomycete and one diatom species (Figure 1, Table 1 and Additional file 1). CryptonS elements do not encode any GCR1_C domain, but the C-terminal region is conserved among CryptonS elements. CryptonS elements are associated with 5- or 6bp terminal direct repeats. The majority of CryptonS elements share TATGG termini. Some CryptonS elements encode an additional protein containing a C48 peptidase domain. The peptidases encoded by CryptonS and CryptonF elements in oomycetes belong to the same family and are related to the $U l p 1$ protease family. Domain shuffling between two groups of Crypton elements could explain the similarity, but more data are needed to determine the relationship between these peptidases and other cellular peptidases.

\section{Cryptons in animals (CryptonA and Cryptonl groups)}

We identified Cryptons in seven metazoan animals belonging to five phyla (Table 1 and Additional file 1). CryptonI elements were found only in insects, whereas CryptonA elements were found in various animals, including cnidarians. Animal Cryptons (both CryptonA and CryptonI) have no C-terminal domain (Figure 1). We did not find any terminal repeats in animal Cryptons. CryptonI-1_RPro from Rhodnius prolixus hosts a non-autonomous derivative family, CryptonI-1N1_RPro, in which 5' 438 bp and 3' 260 bp are $98 \%$ identical to those of CryptonI-1_RPro. This is the first report of non-autonomous Crypton elements. Comparison of 50 copies of CryptonI-1_RPro and CryptonI-1N1_RPro revealed no terminal repeats (neither direct nor inverted). In medaka, we also found two families of non-autonomous derivatives (CryptonA-1N1_OL and CryptonA-1N2_OL) of CryptonA-1_OL. As in the case of other DNA transposons, Crypton non-autonomous elements are much more abundant than their autonomous counterparts.

We can safely rule out the theoretically possible contamination of the genomic sequences from medaka used in this study. First, we identified more than 2,700 copies of autonomous and non-autonomous Crypton elements with DNA sequence identities to consensus ranging from 59\% to $98 \%$. The nucleotide diversity of Cryptons from medaka is consistent with their long-term presence in the medaka lineage. Second, we found many Crypton sequences in the database of expressed sequence tags (ESTs) from three different medaka strains: Hd-rR, CAB and HNI (data not shown). We also found several Cryptons with inserted medaka-specific transposons such as piggyBac-N1_OL and RTE-1_OL (Table 2).

\section{Crypton-derived sequences in the ATF7IP gene}

Identification of Cryptons in three deuterostome species (medaka, sea urchin and acorn worm) prompted us to extend analysis of Cryptons in chordates, including four sequenced actinopterygian species (Fugu rubripes, Tetraodon nigroviridis, Gasterosteus aculeatus and Danio rerio). Although multiple copies of Crypton elements were found only in medaka, sequences similar to Cryptons were found in various chordate species (Table 3). Most of them do not encode any functional recombinases, owing to frameshifts, deletions and substitutions at catalytically essential residues.

However, two similar sequences (ABQF01015803 from the zebra finch Taeniopygia guttata and AAVX01068049 from the chimaera (elephant shark) Callorhinchus milii) include an intact open reading frame of YR (Figure 4A). We did not further analyze the sequence from chimaera, because the sequenced region was only 2,661 bp in length. The Crypton-like sequence in zebra finch is inside an intron of a gene coding for activating transcription factor 7 interacting protein (ATF7IP) (Figure 4B). There is a YR sequence at the orthologous locus of chicken Gallus gallus, which encodes a protein $97 \%$ identical to that of zebra finch, but it contains a frameshift inside the YR region. The orthologous YR sequence from the turkey Meleagris gallopavo contains a frameshift at the same position (data not shown). Because the divergence between chicken and zebra finch occurred some 107 million years ago (MYA) [30], this unusually high similarity indicates a strong selection operating on these YR sequences. An exon-intron prediction program would predict alternative splicing in the ATF7IP gene from zebra finch, although at present there are no mRNA or ESTs corresponding to the fusion transcript. It is possible that the YR is translated as part of the ATF7IP protein and retains catalytic activity in some birds.

Using the University of California Santa Cruz (UCSC) Genome Browser http://genome.ucsc.edu/, we found that there are partial Crypton sequences at the orthologous positions of the ATF7IP gene from the human, horse, kangaroo and platypus genomes (Figures $4 \mathrm{~B}$ and $4 C)$. There are also closely related sequences present in the genomes of rhesus macaque and tarsier. Therefore, the insertion of Crypton in the ATF7IP gene must have occurred in the common ancestor of amniotes more than 325 MYA [30]. None of the mammalian orthologous sequences encode intact YR proteins, and many mammalian species are missing the YR sequence. This indicates only a slight, if any, selective pressure on this sequence in mammals.

\section{Ancient domestication of Cryptons in animals}

Most vertebrate genes similar to Crypton code for proteins (Additional file 4). In the human genome, there are 
Table 2 Crypton copies containing insertions of other transposable elements

\begin{tabular}{|c|c|c|c|c|c|c|c|}
\hline Chromosome & Start $^{a}$ & End $^{a}$ & Element & Start $^{b}$ & End $^{b}$ & Direction $^{c}$ & Identity $^{d}$ \\
\hline \multirow[t]{3}{*}{ chr1 } & 35100344 & 35100443 & Crypton-1N1_OL & 470 & 573 & c & 0.8614 \\
\hline & 35100444 & 35100648 & piggyBAC-N1_OL & 1 & 204 & $d$ & 0.9512 \\
\hline & 35100649 & 35101118 & Crypton-1N1_OL & 1 & 474 & $c$ & 0.8728 \\
\hline \multirow[t]{3}{*}{ chr23 } & 4844226 & 4844361 & Crypton-1N1_OL & 1 & 147 & $d$ & 0.9489 \\
\hline & 4844362 & 4844566 & piggyBAC-N1_OL & 1 & 205 & $d$ & 0.9854 \\
\hline & 4844567 & 4844980 & Crypton-1N1_OL & 144 & 570 & $d$ & 0.9294 \\
\hline \multirow[t]{3}{*}{ chr23 } & 7640079 & 7640510 & Crypton-1N1_OL & 1 & 441 & $d$ & 0.8918 \\
\hline & 7640511 & 7640716 & piggyBAC-N1_OL & 1 & 205 & $d$ & 0.9466 \\
\hline & 7640717 & 7640832 & Crypton-1N1_OL & 438 & 573 & $d$ & 0.8814 \\
\hline \multirow[t]{3}{*}{ chr5 } & 2033028 & 2033143 & Crypton-1N1_OL & 3 & 116 & $d$ & 0.8966 \\
\hline & 2033144 & 2033348 & piggyBAC-N1_OL & 1 & 205 & c & 0.9659 \\
\hline & 2033349 & 2033803 & Crypton-1N1_OL & 113 & 573 & $d$ & 0.9132 \\
\hline \multirow[t]{3}{*}{ chr5 } & 22193852 & 22194261 & Crypton-1N1_OL & 144 & 573 & c & 0.9030 \\
\hline & 22194262 & 22194466 & piggyBAC-N1_OL & 1 & 205 & $d$ & 0.9707 \\
\hline & 22194467 & 22194605 & Crypton-1N1_OL & 1 & 147 & c & 0.9078 \\
\hline \multirow[t]{3}{*}{ chr8 } & 7666866 & 7667209 & Crypton-1N1_OL & 21 & 376 & $d$ & 0.9075 \\
\hline & 7667210 & 7667896 & RTE-1_OL & 2,666 & 3,352 & c & 0.9796 \\
\hline & 7667897 & 7668065 & Crypton-1N1_OL & 372 & 550 & $d$ & 0.8667 \\
\hline
\end{tabular}

${ }^{\mathrm{a}}$ Sequence coordinates in the corresponding chromosome. ${ }^{\mathrm{b}}$ Corresponding coordinates of the consensus sequences of repeat elements from Repbase. ${ }^{\mathrm{c}}$ Sequence orientation relative to consensus: (d)irect and (c)omplementary orientation. ${ }^{\mathrm{d}}$ Identity to the consensus sequences.

seven proteins similar to Crypton YRs, which are annotated as parts of six genes (Figure 5 and Additional file 5). The KIAA1958 gene contains two isoforms, both of which include YR-derived sequences. The other genes are potassium channel tetramerization domain containing 1 (KCTD1), zinc finger, myeloproliferative and mental retardation type 2 (ZMYM2)/zinc finger protein 198 (ZNF198), ZMYM3/ZNF261, ZMYM4/ZNF262 and glutamine-rich protein 1 (QRICH1) (Figure 5). A PSIBLAST search of these proteins against the National Center for Biotechnology Information (NCBI) conserved domain database (CDD) revealed that they share a domain of unknown function (DUF3504 superfamily; $E$-value $\leq 1 \mathrm{e}-29)$. The six genes are widespread among vertebrates (Figure 6) and are highly conserved among phylogenetically distant species (Table 4). The phylogenetic relationship of each gene agreed with that of species (data not shown). The nucleotide sequences corresponding to all seven DUF3504 domains were present in the NCBI EST database, indicating their expression. The data clearly show that they are neither pseudogenes nor defective Cryptons (see the accession numbers of DUF3504 genes in Additional file 5). However, none of them preserve the YR catalytic site. All of them lost the catalytic tyrosine and the second conserved arginine, and all but KCTD1 also lost the conserved histidine.

Although the resolution is low because of high divergence and the short length of the YR sequence, animal DUF3504 genes tend to cocluster with animal Cryptons (CryptonA) in the YR phylogenetic tree (Figure 2). There are four independent clusters of DUF3504 genes: KCTD1, KIAA1958a, KIAA1958b/KIAA1958L and WOC/ ZMYM/QRICH1 (Figure 2, filled circles). KCTD1 coclusters with several animal Cryptons, and the clustering is supported by $100 \%$ bootstrap value. Cryptons form a paraphyletic cluster, which indicates that the DUF3504

Table 3 Molecular fossils of Cryptons in chordates

\begin{tabular}{ll}
\hline Species & Accession numbers \\
\hline Danio rerio & BX530066* \\
Xenopus tropicalis & NP_001120376, AAMC01135377*, AAMC01082917* \\
Callorhinchus milii & AAVX01521991*, AAVX01068049*, AAVX01132927* \\
Ciona intestinalis & XP_002124034, XP_002125964 \\
Ciona savignyi & AACT01002283*, AACT01041791* \\
Halocynthia roretzi & BAB40645 \\
Oikopleura dioica & CBY34656 \\
Branchiostoma floridae & XP_00260067, XP_002595788, XP_002613958,XP_002613959, XP_002587732, XP_002607491 \\
\hline
\end{tabular}

*Nucleotide sequences including Crypton fragments. 


\section{A \\ ATF7P_Chicken \\ ATF7P-ZZebrafinch
AAVXo1068049 \\ Crypton-1.1.SP \\ $C$ CryptonA-2.SP
CryptonA-1_SK \\ CryptonA-2_SK
CryptonA-3 SKK
CryptonA-1_OLL}
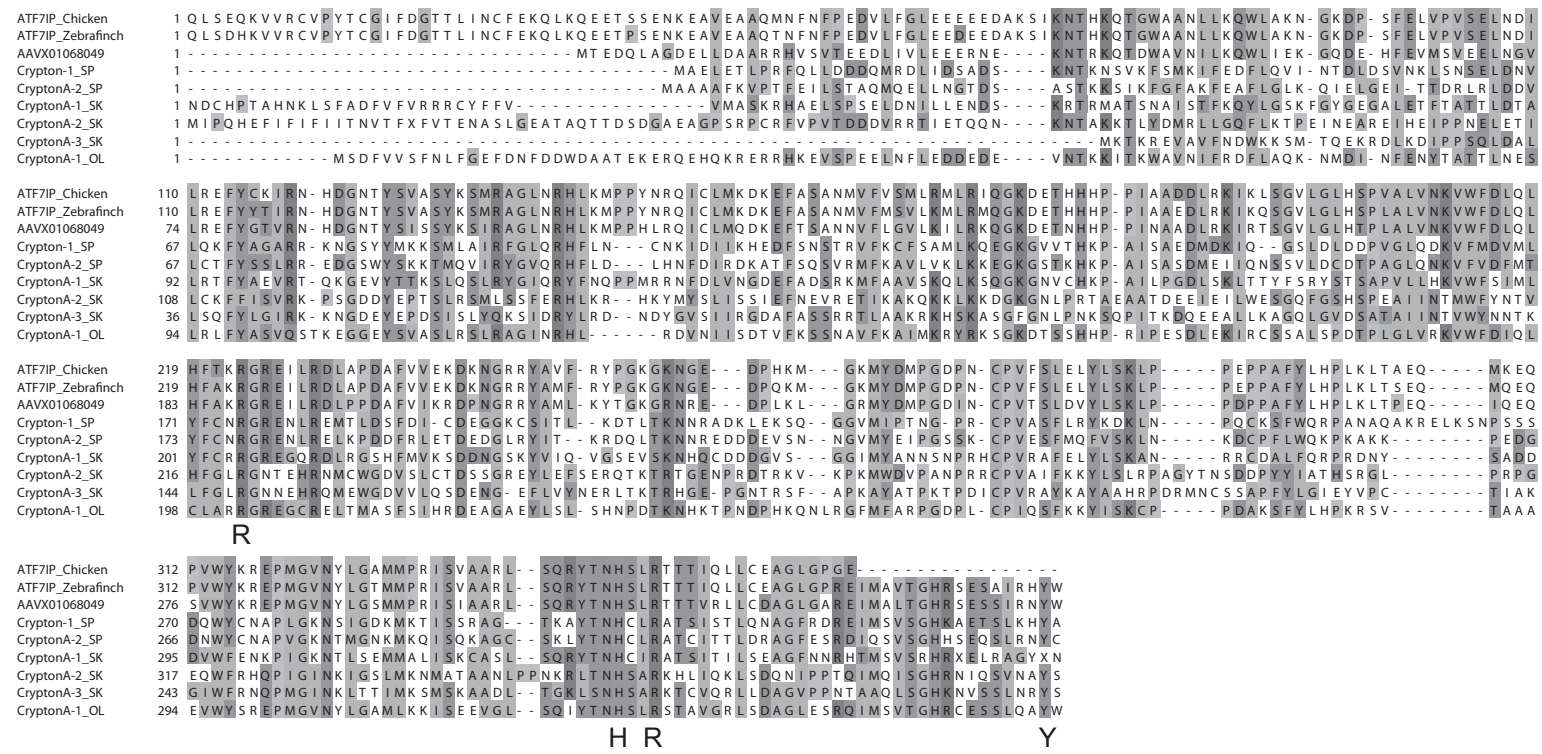

B

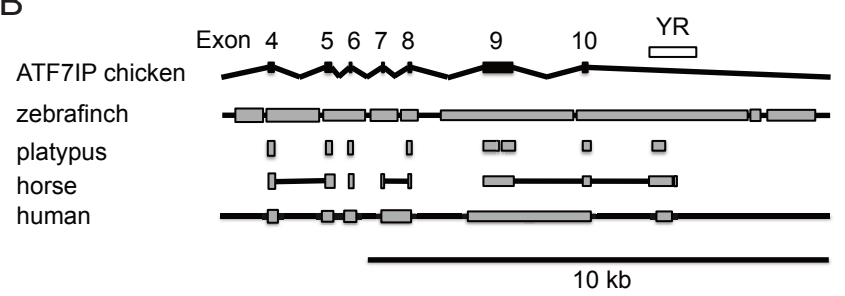

C

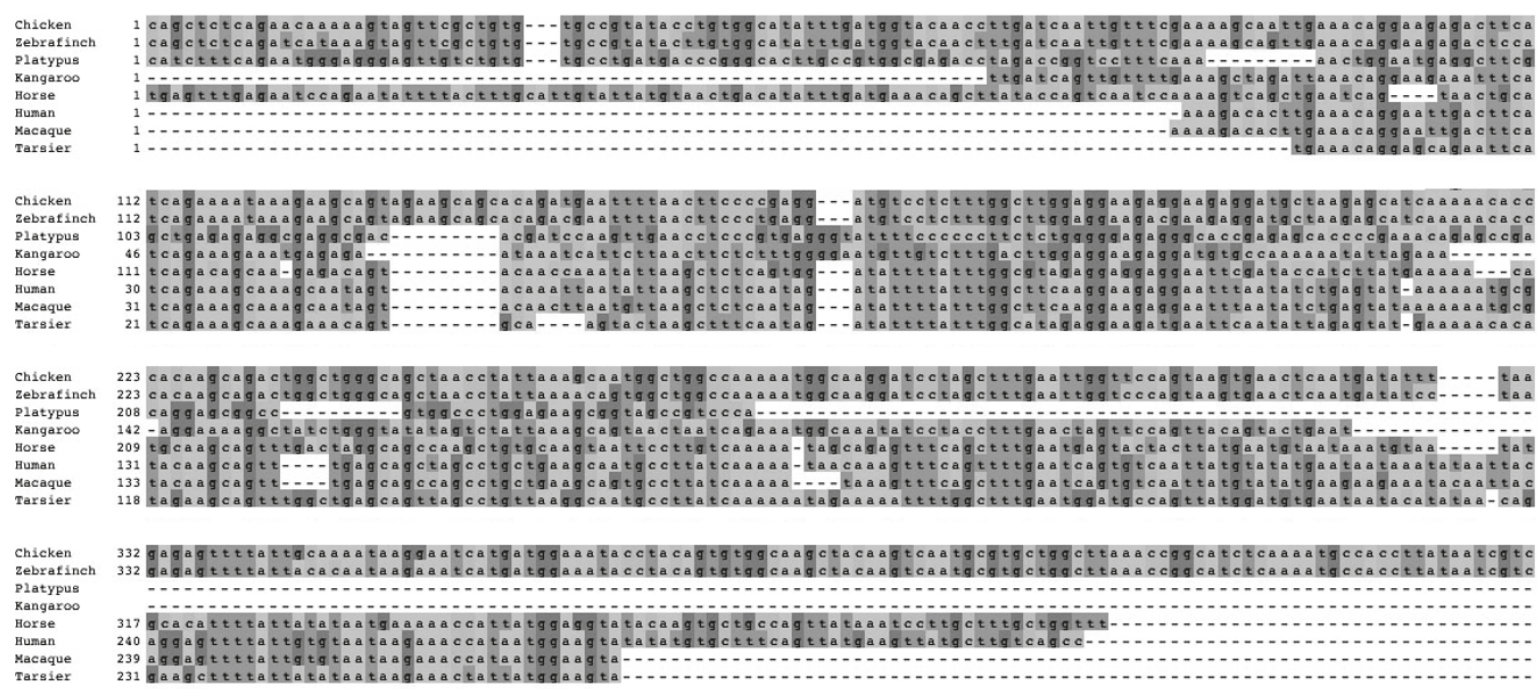

Figure 4 Crypton-derived sequence in an intron of ATF7IP gene. (A) Alignment of proteins coded by deuterostome Cryptons and Cryptonderived sequences. Catalytically essential residues are shown below the alignment. (B) Illustration of the conservation of ATF7IP loci. The position of the YR sequence is indicated by the open box. Black boxes represent exons of the chicken ATF7IP gene. Gray boxes indicate conserved blocks between chicken and respective species based on the Net Tracks of the UCSC Genome Browser http://genome.ucsc.edu/. Lines between gray boxes indicate that boxes are connected by unalignable sequences. (C) Alignment of nucleotide sequences of Crypton-derived sequences. 


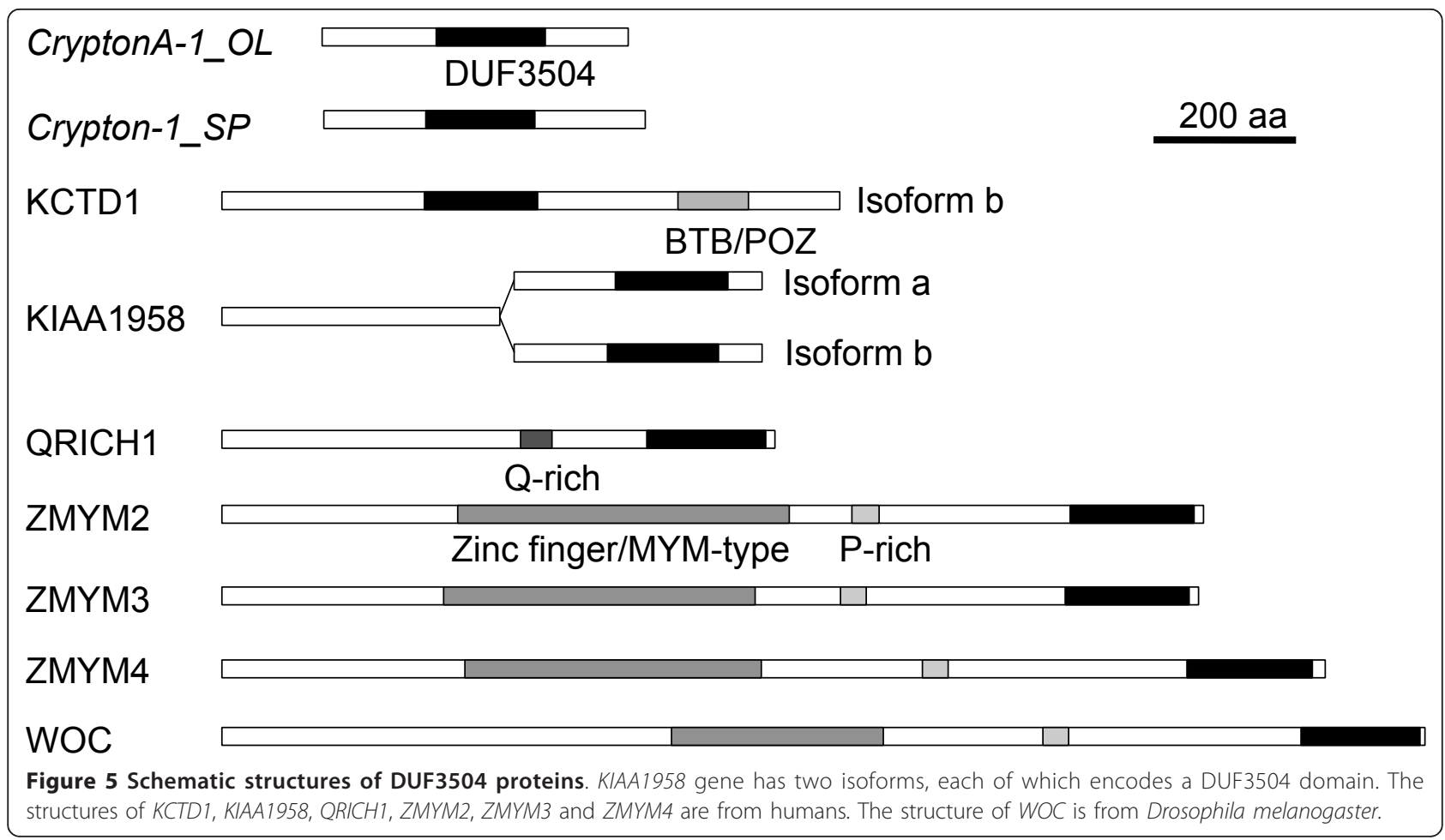

domain of KCTD1 was derived from a Crypton YR. The position of KIAA1958a is distinct from either CryptonA or CryptonI, and WOC/ZMYM/QRICH1 is clustered as a sister group of all animal CryptonA elements. Therefore, phylogeny alone does not support the domestication of animal Cryptons leading to WOC/ZMYM/QRICH1 and KIAA1958a.

The DUF3504 domain was derived from YR, not vice versa, because DUF3504 lacks the complete catalytic tetrad essential for YR activity. YR is essential for transposition, and repeated generation of active YRs from defective YRs is highly improbable. The distributions of WOC/ZMYM/ QRICH1 and KIAA1958a are restricted to bilaterians and jawed vertebrates, respectively. Apart from Cryptons, the only other possible sources of YRs in animal genomes are the retrotransposon families DIRS and Ngaro [23,24]. However, all searches of the YRs from CryptonA-1_OL, Crypton-1_SP and CryptonA-1_SK matched the DUF3504 sequence with $E$-values $\leq 8 \mathrm{e}-12$, whereas YRs of DIRS and Ngaro did not match the DUF3504 sequence at all (even when the threshold $E$-value was set at 100$)$. Several representatives of DUF3504 are actually Crypton sequences; for example, XP_001639277 is the protein coded by Crypton$1 \_N V$. The patchy distribution of Cryptons and the inconsistency between Crypton and host phylogenies indicate ancient amplification and extinction events in Crypton evolution. The ancient amplifications would have generated many lineages of Cryptons, and it is likely that $\mathrm{WOCl}$ ZMYM/QRICH1 and KIAA1958a derived from lineages of
Cryptons that are now extinct. We cannot completely rule out the possibility that the two genes and CryptonA elements were independently derived from DIRS-like retrotransposons or some as yet uncharacterized types of mobile elements, but this implies independent origins of CryptonA and other Crypton groups (CryptonF, CryptonS and CryptonI). Therefore, four independent domestication events of animal Cryptons are the most parsimonious explanation for the origins of animal DUF3504 genes.

A representative of DUF3504 from Halocynthia roretzi (BAB40645) has orthologs in other tunicates: Ciona intestinalis (XP_002125964), Ciona savignyi (AACT01002283 and AACT10141791) and Oikopleura dioica (CBY34656). They could also represent a domestication event of Crypton. Another representative (YP_025778) is coded in the mitochondrion of the green alga Pseudendoclonium akinetum. It could also be a candidate Crypton-derived gene; owing to the lack of related sequences, however, we did not analyze it further.

All DUF3504 genes encode much longer proteins than their DUF3504 domains, and it is possible that the preexisting genes captured entire Crypton protein-coding sequences. However, the only recognizable domain encoded by animal Cryptons is YR (DUF3504), and there is little sequence similarity beyond YRs among Cryptons themselves. Therefore, it is unlikely that any significant sequence similarity was preserved beyond the DUF3504 domains between the DUF3504 and Crypton proteins. 


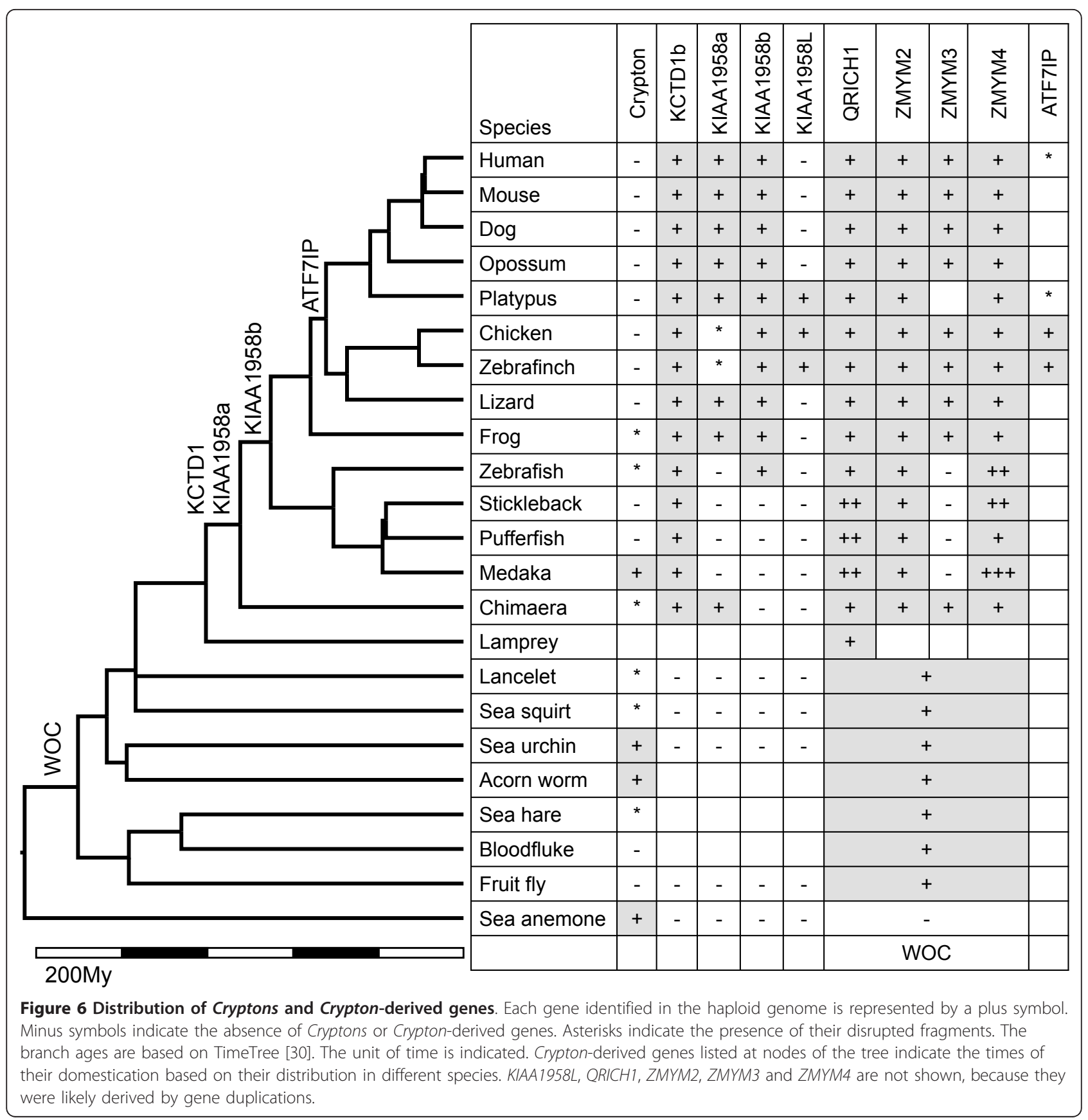

\section{KCTD1 gene}

The KCTD1 gene contains the DUF3504 domain confined within a single exon. Among the vertebrate genes, the KCTD1 DUF3504 domain is the closest to the Crypton YRs in terms of protein sequence similarity. The sequence identity between the KCTD1 DUF3504 domain

Table 4 Protein identities between DUF3504 domains in two species

\begin{tabular}{|c|c|c|c|c|c|c|}
\hline Comparison & KCTD1 & KIAA1958b & QRICH1 & ZMYM2 & ZMYM3 & ZMYM4 \\
\hline Human-chicken & $98 \%$ (268 of 274$)$ & $99 \%$ (282 of 287 ) & 97\% (299 of 309) & $92 \%$ (287 of 313 ) & $85 \%(265$ of 315$)$ & $88 \%$ (302 of 347) \\
\hline Human-zebrafish & $90 \%$ (273 of 304$)$ & $86 \%$ (245 of 287 ) & $75 \%$ (229 of 306$)$ & $47 \%$ (145 of 310$)$ & - & $\begin{array}{l}52 \%(\text { ZMYM4a) } \\
(176 \text { of } 345) 52 \%(\text { ZMYM4b) } \\
\text { (169 of 331) }\end{array}$ \\
\hline
\end{tabular}


and the YR of Crypton-1_SP is 32\%, which exceeds the analogous identity among different lineages of Cryptons. For example, Crypton-1_SP in the CryptonA lineage and Crypton-1_TC in the CryptonI lineage show less than $30 \%$ sequence identity to each other. KCTD1 encodes two protein isoforms of different lengths. The longer isoform (isoform b) contains both an $\mathrm{N}$-terminal DUF3504 domain and a C-terminal BTB/POZ (Broadcomplex, Tramtrack and Bric-a-brac/poxvirus and zinc finger) domain (Figure 5), whereas the shorter one (isoform a) contains only the $\mathrm{BTB} / \mathrm{POZ}$ domain. The shorter isoform is approximately $80 \%$ identical to the KCTD15 gene at the protein level, and related genes are found in various organisms, including lancelet, sea urchin and insects (Additional file 6). The KCTD15 gene does not have any DUF3504 domain and is found in gnathostomes from mammals to chimaera. We infer that KCTD1 and KCTD15 were duplicated from a single gene in the early evolution of vertebrates and after that a Crypton copy was inserted upstream of the KCTD1 gene, which generated a new transcriptional variant encoding the isoform $b$. This insertion should have occurred before the branching of the Chondrichthyes (sharks, rays, skates and chimaeras) about 527 MYA [30].

\section{KIAA1958 gene}

The KIAA1958 gene, of unknown function, is present in two isoforms ( $\mathrm{a}$ and $\mathrm{b}$ ) which contain different DUF3504 domains encoded by different exons (Figure 5). DUF3504 domains in isoforms a and b are only $26 \%$ identical to each other in the human genome. Although alternative splicing has not been confirmed experimentally, the high conservation of both DUF3504-coding sequences indicates that both encode functional proteins (Table 4 and Additional file 4). Neither of the two DUF3504-coding sequences is interrupted by introns. We found both isoforms in a wide range of tetrapods (Figure 6). Zebrafish lacks isoform a, whereas Chondrichthyes (chimaera) lack isoform b. Some Actinopterygii (medaka, stickleback, fugu and pufferfish) lack both isoforms. Chicken, zebra finch and platypus have an additional gene similar to KIAA1958 isoform b, designated KIAA1958L. The exons encoding isoform-specific regions of KIAA1958b are positioned upstream from those of KIAA1958a. The KIAA1958L gene likely originated from a duplication of the segment including KIAA1958b-specific exons but not including KIAA1958a-specific exons, and this duplication event predated the branching between mammals and birds. KIAA1958L is less conserved than KIAA1958 isoforms a and b. The DUF3504 domains of KIAA1958L proteins from platypus and chicken are only $44 \%$ identical, whereas those of the KIAA1958b are $97 \%$ identical between the species. KIAA1958a is nonfunctional in chicken and zebra finch, but it is intact in lizard. Isoform b was not found in Chondrichthyes, and it is possible that it originated later in the lineages which branched off Chondrichthyes. KIAA1958a might have originated in the common ancestor of gnathostomes. Another possibility is that both isoforms were acquired in the common ancestor of gnathostomes and isoforms $\mathrm{a}$ and $\mathrm{b}$ had been lost in the lineages of Actinopterygii and Chondrichthyes, respectively.

\section{ZMYM2, ZMYM3, ZMYM4 and QRICH1 genes}

The $Z M Y M 2, Z M Y M 3$ and $Z M Y M 4$ genes are present in diverse gnathostomes from human to chimaera (Figure 6). $Z M Y M 2, Z M Y M 3$ and ZMYM4 are similar to arthropod $W O C$ in terms of their sequence and structure $[31,32]$. The DUF3504 domains from the Drosophila melanogaster WOC gene and the human ZMYM2 gene are $41 \%$ identi$\mathrm{cal}$ at the protein level. Some introns are also positioned at the corresponding sites of ZMYM2 and WOC (Figure 7A). In addition to chordates and arthropods, we found sequence fragments related to $W O C$ in echinoderms (Strongylocentrotus purpuratus), hemichordates (Saccoglossus kowalevskii), mollusks (Pinctada maxima and Aplysia californica) and platyhelminthes (Schistosoma mansoni, S. japonicum and Schmidtea mediterranea) (Additional file 5). There is no evidence that $W O C$ forms multiple gene families in invertebrates. The ZMYM2, ZMYM3 and $Z M Y M 4$ genes are listed in the data set of ohnologs reported recently by Makino and McLysaght [33], which means that they were duplicated from a single gene during two rounds of whole-genome duplication in the early evolution of vertebrates before the split between jawed vertebrates and agnathans [34,35]. The synteny blocks of $Z M Y M 2, Z M Y M 3$ and ZMYM4 share several genes in addition to $Z M Y M$ genes (Figure $7 \mathrm{~B}$ ). The most parsimonious scenario is that the $W O C / Z M Y M$ gene family originated from the domestication of Crypton in the common ancestor of bilaterians.

There are three other ZMYM genes (ZMYM1, $Z M Y M 5$ and ZMYM6) in the synteny blocks (Figure $7 \mathrm{~B})$, but they have no DUF3504 domain. The N-terminal part of ZMYM5 is similar to that of ZMYM2, whereas those of ZMYM1 and ZMYM6 are similar to that of ZMYM4. These three genes are present only among eutherian mammals. These data support independent gene duplication events inside each synteny block. It is noteworthy that the C-terminal parts of ZMYM1, ZMYM5 and ZMYM6 derived from transposases of $h A T$-type DNA transposons, but these $h A T$ derived sequences are not close to each other. The C-terminal part of ZMYM6 is close to Charlie elements in the human genome, whereas that of ZMYM1 is closer to plant $h A T$ elements such as HAT-1_Mad from apple (data not shown). 


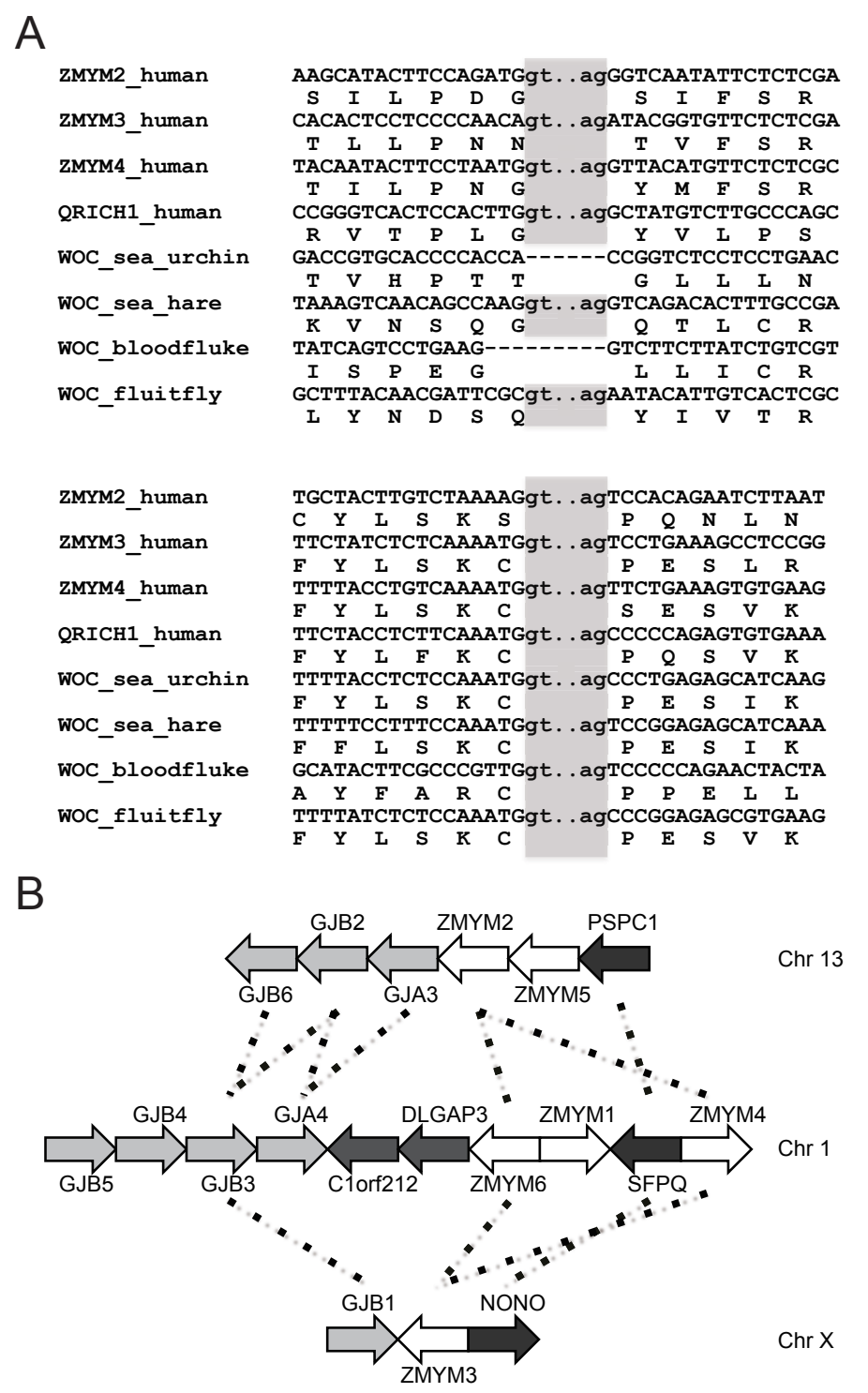

Figure 7 Paralogous relationships of WOC/ZMYM/QRICH1 genes. (A) Two conserved intron positions among WOC, ZMYM2, ZMYM3, ZMYM4 and $Q R I C H 1$. Introns are printed in lowercase letters and shaded. Protein sequences are shown below nucleotide sequences. The upper and lower intron positions correspond to the 20th and 22nd introns of human ZMYM2, respectively. (B) The synteny blocks of ZMYM2, ZMYM3 and ZMYM4. Ohnologous relationships reported by Makino and McLysaght [33] are indicated by dotted lines. GJB = gap junction protein $\beta ; G J A=$ gap junction protein $\alpha$; DLGAP3 = discs large homolog-associated protein 3; C1orf212 = chromosome 1 open reading frame 212. Other gene names are described in the text.

The QRICH1 gene was found in diverse vertebrates, including lamprey (Figure 6). The DUF3504 domain in QRICH1 is quite similar to those of ZMYM2, ZMYM3 and ZMYM4. Besides, five of eight introns of QRICH1 were at the sites corresponding to those of $Z M Y M 2$, ZMYM3 and ZMYM4 (Figure 7A and data not shown). The high structural and sequence similarity between WOC, ZMYM2/3/4 and QRICH1 indicates that QRICH1 originated from either $W O C$ or $Z M Y M$ genes. In the neighborhood of QRICH1, we could not find any genes paralogous to genes in the synteny blocks of ZMYM2,
ZMYM3 and ZMYM4. However, because QRICH1 is present in the lamprey genome, it must have originated at the time close to the whole-genome duplication events.

\section{Discussion}

\section{Evolution of WOC: the third-oldest event of transposon domestication}

The most ancient transposon-derived gene known to date is TERT, which was generated by the domestication of a Penelope-like retroelement [4], and $\operatorname{Prp} 8$, a spliceosomal 
component derived from a retrointron (group II self-splicing intron) [36]. TERT retains the catalytic activity of RT, but Prp8 does not. These two genes are shared by almost all eukaryotes. Another example of an ancient domestication event is the $R A G 1$ gene [5]. It is distributed widely among gnathostomes, but no RAG1 ortholog was found in agnathans, including lamprey and hagfish. Given that agnathans have a different type of adaptive immune system called "variable lymphocyte receptors" [37], the domestication of RAG1 likely occurred in the last common ancestor of gnathostomes after their branching from agnathans. Other transposons domesticated in the distant past are in HARBI1 and PBDG5 genes, both of which are present in vertebrates from humans to actinopterygian fish [38,39]. The KCTD1b, KIAA1958a and KIAA1958b genes are as old as or older than the HARBI1 and PBDG5 genes (Figure 6). A transposon-derived CENP-B, a highly conserved mammalian centromere, and three CENP-B-like proteins (Abp1, Cbh1 and $\mathrm{Cbh} 2$ ) in fission yeast resemble each other in terms of their sequences and functions, but they derived independently from different pogo-like transposases [40]. The human genome harbors a significant number of genes derived from transposons [6]. Some of them were domesticated in the distant past, and there are no traces of related repetitive sequences or TEs from which they were derived. For example, the HARBI1 gene was derived from PIF/Harbinger and PHSA (THAP domain-containing protein 9 , or THAP9) from a $P$-like element $[38,41]$. Both HARBI1 and PHSA were found by screening mammalian genes against DNA transposons from zebrafish. Similarly, the key to our findings of Crypton-derived genes was screening of genes against Cryptons preserved in medaka, because there are only a few remnants of Cryptons left in vertebrate genomes sequenced to date, except in medaka.

The ancestral gene for $W O C / Z M Y M$ probably originated in the common ancestor of all bilaterians more than 910 MYA [30]. This is the third-oldest transposon domestication event known to date, following the two domestication events of RT $[4,36]$. Our study indicates that domestication of Crypton-like elements in eukaryotes was relatively common in the distant past. This implies that Cryptons are very ancient and, given their rare occurrence in the genomic fossil record and their great diversity, they were probably much more active in the distant past than in more recent evolutionary history.

\section{Functional implications for domesticated Crypton YRs}

No function of DUF3504 domains has been reported to date. Even so, the YR origin of DUF3504 domains implies their functions to some extent. YR forms a multimer when it binds substrate DNA during recombination [42]. On that basis, we can envision two possible functions derived from YRs: DNA binding and protein-protein interaction.
There are several indications for functions of domesticated YRs. First, many genes derived from YRs are transcriptional regulators. Gcr1, KCTD1, WOC, ZMYM2, ZMYM3 $Z M Y M 4$, and $A T F 7 I P$ are either transcriptional activators or repressors [43-48]. Cbf2 acts as a centromeric protein directly binding to centromere-specific sequences and is essential for spindle pole body formation [49,50]. Although these proteins usually contain a DNA-binding domain other than DUF3504, exemplified by the GCR1_C domain of Gcr1 and Cbf2, the DUF3504 domain could also work as a DNA-binding domain. Second, there is an interesting resemblance between functions of domesticated DDEtransposases and YRs. Daysleeper is a transcription factor derived from a $h A T$ DDE-transposase and binds a specific motif for transcription regulation [12]. CENP-B is a centromere protein derived from the DDE-transposase of a pogo-like transposon [13]. In these genes, transposasederived domains act as DNA-binding domains. Third, a large family of prokaryotic transcriptional activators, AraC/XylS, shows structural similarity to YRs. The overall fold of the 129-amino acid protein MarA, a member of the $\mathrm{AraC} / \mathrm{XylS}$ family, almost entirely recapitulates the YR domain of Cre recombinase [51]. MarA can simultaneously bind RNA polymerase II and DNA to form a ternary complex [52]. These data support the putative function of DUF3504 to be DNA or protein binding.

To date relatively little is known about Cryptons. There have been no studies of their transposition, transcription, translation or regulation. The sequence similarity between Cryptons is very low, especially in their non-protein-coding regions. We compared DNA sequences of Cryptons from different species, but we could not find any conserved nucleotide sequences among them. Furthermore, all Crypton domestication events are very old. Therefore, it is very difficult to propose any specific functions of DUF3504 domains. Instead, herein we propose potential pathways in which DUF3504 domains could be involved.

KCTD 1 and KCTD15 are paralogs that have diverged during the early evolution of vertebrates (Additional file 6). KCTD1 isoform b, generated by an insertion of Crypton upstream of the original KCTD1 gene, is widely conserved among jawed vertebrates (Figure 6), although it is unclear whether the agnathans carry the $K C T D 1 b$ gene. The high conservation of KCTD1b (Table 4) indicates its essential function shared among jawed vertebrates. KCTD1 represses the activity of the AP- $2 \alpha$ transcription factor, and the $\mathrm{BTB} / \mathrm{POZ}$ domain is responsible for the interaction [46]. AP- $2 \alpha$ plays an essential role in neural border (NB) and neural crest (NC) formations during embryonic development [53]. NB is the precursor of NC. KCTD15 is expressed in NB and inhibits NC induction [54]. The NC cells are a transient, multipotent, migratory cell population unique to vertebrates. They give rise to diverse cell lineages. We can speculate that by adding a 
new protein-protein or protein-DNA interaction $K C T D 1 b$ can contribute to the network of NC formation through the regulation of AP- $2 \alpha$.

Among DUF3504 genes, the function of WOC/ZMYM is of special interest because two of the genes in this group, $Z M Y M 2$ and $Z M Y M 3$, are linked to human diseases. A chromosomal translocation between ZMYM2 and fibroblast growth factor receptor 1 (FGFR1) causes lymphoblastic lymphoma and a myeloproliferative disorder [55]. A translocation involving ZMYM3 is associated with $\mathrm{X}$-linked mental retardation [56]. Mutations of their ortholog, WOC, cause larval lethality in D. melanogaster [31].

WOC/ZMYM gene-encoded proteins are involved in various processes, including transcription, DNA repair and splicing. WOC is a transcriptional regulator that colocalizes with the initiating forms of RNA polymerase II $[31,32]$. The WOC proteins also colocalize with all telomeres, and mutants of $W O C$ are associated with frequent telomeric fusions [31,32]. ZMYM2, ZMYM3 and ZMYM4 are components of a multiprotein corepressor complex, including histone deacetylase 1 (HDAC1) and HDAC2 $[47,48]$. ZMYM2 binds to various transcriptional regulators including Smad proteins [57]. It also binds to proteins involved in homologous recombination, such as RAD18, HHR6A and HHR6B, which are human orthologs of the yeast RAD proteins [58], and to spliceosomal components including SFPQ (splicing factor, proline- and glutaminerich) [59].

Interestingly, the SFPQ gene is a component of the syntenic cluster of ZMYM4 (Figure 7B). The paralog of SFPQ in the cluster of ZMYM3 is NONO (non-POU domaincontaining, octamer-binding), which is a partner of SFPQ in heteromers [60]. PSPC1 (paraspeckle component 1) present in the cluster of ZMYM2 also shows similarity to $S F P Q$ and NONO genes. In addition to their involvement in splicing, the SFPQ proteins contribute to DNA repair by interacting with RAD51 [61]. They are also recruiting HDAC1 to the STAT6 transcription complex [62]. Therefore, it is likely that WOC/ZMYM and SFPQ/NONO/ PSPC1 proteins cooperatively act in transcription regulation, splicing and DNA repair, and that they have coevolved by maintaining their functional relationships. Their DUF3504 domains may contribute to some of the proteinprotein interactions.

\section{Evolution of Cryptons}

To date Cryptons have been identified in a limited number of fungi and animal species. Herein we report the presence of Cryptons in new species, but information regarding their overall distribution continues to be patchy (Table 1). CryptonF elements are present in three phyla of fungi (Ascomycota, Basidiomycota and
Zygomycota) and two orders of oomycetes (Peronosporales and Saprolegniales). Our phylogenetic analysis supports the horizontal transfer of CryptonF elements between fungi and oomycetes (Figure 2), which is consistent with frequent horizontal transfer of genes between them [63]. CryptonS elements are also present in two oomycete orders and one species of diatoms. Both oomycetes and diatoms are lineages of stramenopiles, and the origin of CryptonS elements could date back to their common ancestor.

Animal Cryptons (CryptonA and CryptonI) were found in six phyla: Chordata, Echinodermata, Hemichordata, Arthropoda, Mollusca and Cnidaria. CryptonI elements have the same overall structure as CryptonA elements and were observed only in insect genomes. It is possible that CryptonI elements constitute a branch of CryptonA but they have evolved more rapidly in insects. The overall distribution in fungi, oomycetes and animals indicates that Cryptons were long present in these three eukaryotic groups, probably with some contribution of a horizontal transfer. It is likely that Cryptons originated in the common ancestor of these three groups, although because of the low resolution of the YR phylogeny, we cannot rule out the possibility of their independent origins.

The identification of Crypton elements in medaka is surprising. The nucleotide diversity of Cryptons in the medaka genome clearly shows that Cryptons were maintained in the lineage leading to medaka for a long time. It is possible that Cryptons invaded the medaka population after the split of medaka from the three actinopterygian fish species (Gasterosteus, Takifugu and Tetraodon), whose genomes have been sequenced. The vertical transfer of Cryptons in the lineage leading to medaka is a preferable scenario because of the domestication of Crypton in the common ancestor of bilaterian animals, which led to the origin of WOC genes. In most identified host organisms, Cryptons are preserved in very low copy numbers (Additional file 1). We found several fragments of Cryptons in various vertebrates, including zebrafish (Table 3). The origin of Cryptonderived genes took place at different times during the evolution of vertebrates (Figure 6). This is consistent with the hypothesis that Cryptons continued to maintain very low copy numbers in the vertebrate genomes and were occasionally amplified in certain lineages.

\section{Conclusions}

This study has revealed the diversity of a unique class of DNA transposons, Cryptons, and their repeated domestication events. The DUF3504 domains are domesticated YRs of animal Crypton elements. Our findings add a new repertoire of domesticated proteins and provide 
further evidence for an important role of transposable elements as a reservoir for new cellular functions.

\section{Methods}

Data source

Genome sequences of various species were obtained mostly from GenBank, and sequences of known Cryptons, DIRS and Ngaro were obtained from Repbase http://www.girinst.org/repbase/. All characterized Cryptons have been deposited in Repbase.

\section{Sequence analysis}

Characterization of new Cryptons was achieved by repeated BLAST [64] and CENSOR [65] searches using genome sequences of various species with Cryptons as queries. All analyses were done with default settings. The consensus sequences of elements were derived using the majority rule applied to the corresponding sets of multiple aligned copies of Cryptons. Alignment gaps were manually adjusted to maximize similarity to other related elements. Characterization of DUF3504 genes was performed by BLAST searches against both protein and nucleotide databases with known DUF3504 genes as queries. We predicted exon-intron boundaries with the aid of SoftBerry FGENESH:

http://linux1.softberry.com/berry.phtml?topic=fgenesh\&group $=$ programs\&subgroup $=$ gfind and manually adjusted them through the comparison to orthologous sequences in other species.

\section{Sequence alignment and phylogenetic analysis}

We used MAFFT [66] with the linsi option or MUSCLE [67] with default settings to align both nucleotide and protein sequences of various Cryptons and Crypton-derived proteins. We constructed maximum likelihood trees by using PhyML [68,69] with 100 bootstrap replicates [70] for the amino acid substitution model LG. We also constructed trees with other substitution models, WAG, RtREV and DCMut, and with the Neighbor-joining method, but the resolution did not improve. The tree topology search method was Nearest Neighbor Interchange (NNI), and the initial tree was BIONJ. The phylogenetic trees were drawn with FigTree 1.3.1 software http://tree.bio.ed.ac.uk/software/figtree/.

\section{Additional material}

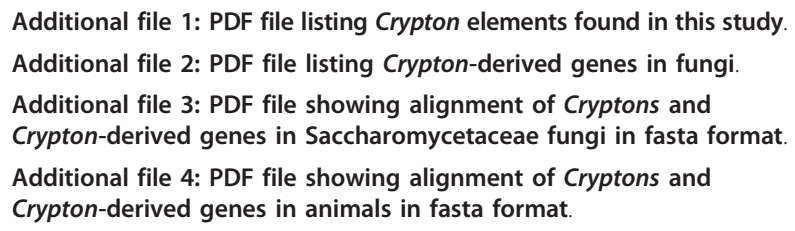

Additional file 5: PDF file listing the accession numbers for DUF3504 genes.

Additional file 6: PDF file showing alignment of KCTD1, KCTD15 and related protein sequences in fasta format.

\section{Abbreviations}

bp: base pair; EN: endonuclease; MYA: million years ago; RT: reverse transcriptase; TE: transposable element; TERT: telomerase reverse transcriptase; TIR: terminal inverted repeat; TSD: target site duplication; YR: tyrosine recombinase.

\section{Acknowledgements}

This work was supported by National Institutes of Health grant 5 P41 LM006252. The content is solely the responsibility of the authors and does not necessarily represent the official views of the National Library of Medicine or the National Institutes of Health.

\section{Authors' contributions}

KKK initiated the research. KKK and JJ performed the research and wrote the manuscript. Both authors read and approved the final manuscript.

\section{Competing interests}

The authors declare that they have no competing interests.

Received: 19 August 2011 Accepted: 19 October 2011

Published: 19 October 2011

\section{References}

1. Kapitonov W, Jurka J: A universal classification of eukaryotic transposable elements implemented in Repbase. Nat Rev Genet 2008, 9:411-414.

2. Miller WJ, Hagemann S, Reiter E, Pinsker W: P-element homologous sequences are tandemly repeated in the genome of Drosophila guanche. Proc Natl Acad Sci USA 1992, 89:4018-4022.

3. Greider $\mathrm{CW}$, Blackburn EH: The telomere terminal transferase of Tetrahymena is a ribonucleoprotein enzyme with two kinds of primer specificity. Cell 1987, 51:887-898.

4. Gladyshev EA, Arkhipova IR: Telomere-associated endonuclease-deficient Penelope-like retroelements in diverse eukaryotes. Proc Natl Acad Sci USA 2007, 104:9352-9357.

5. Kapitonov W, Jurka J: RAG1 core and V(D)J recombination signal sequences were derived from Transib transposons. PLoS Biol 2005, 3:e181.

6. Volff JN: Turning junk into gold: domestication of transposable elements and the creation of new genes in eukaryotes. Bioessays 2006, 28:913-922.

7. Curcio MJ, Derbyshire KM: The outs and ins of transposition: from Mu to kangaroo. Nat Rev Mol Cell Biol 2003, 4:865-877.

8. Aziz RK, Breitbart M, Edwards RA: Transposases are the most abundant, most ubiquitous genes in nature. Nucleic Acids Res 2010, 38:4207-4217.

9. Kapitonov W, Jurka J: Self-synthesizing DNA transposons in eukaryotes. Proc Natl Acad Sci USA 2006, 103:4540-4545.

10. Bao W, Jurka MG, Kapitonov W, Jurka J: New superfamilies of eukaryotic DNA transposons and their internal divisions. Mol Biol Evol 2009, 26:983-993.

11. Bao W, Kapitonov W, Jurka J: Ginger DNA transposons in eukaryotes and their evolutionary relationships with long terminal repeat retrotransposons. Mob DNA 2010, 1:3

12. Bundock P, Hooykaas P: An Arabidopsis $h A T$-like transposase is essential for plant development. Nature 2005, 436:282-284.

13. Tudor M, Lobocka M, Goodell M, Pettitt J, O'Hare K: The pogo transposable element family of Drosophila melanogaster. Mol Gen Genet 1992, 232:126-134.

14. Feng Q, Moran JV, Kazazian HH Jr, Boeke JD: Human L1 retrotransposon encodes a conserved endonuclease required for retrotransposition. Cell 1996, 87:905-916.

15. Kojima KK, Fujiwara $\mathrm{H}$ : An extraordinary retrotransposon family encoding dual endonucleases. Genome Res 2005, 15:1106-1117.

16. Yang J, Malik HS, Eickbush TH: Identification of the endonuclease domain encoded by R2 and other site-specific, non-long terminal repeat retrotransposable elements. Proc Natl Acad Sci USA 1999, 96:7847-7852. 
17. Pyatkov Kl, Arkhipova IR, Malkova NV, Finnegan DJ, Evgenev MB: Reverse transcriptase and endonuclease activities encoded by Penelope-like retroelements. Proc Natl Acad Sci USA 2004, 101:14719-14724.

18. Nunes-Düby SE, Kwon HJ, Tirumalai RS, Ellenberger T, Landy A: Similarities and differences among 105 members of the Int family of site-specific recombinases. Nucleic Acids Res 1998, 26:391-406.

19. Goodwin TJ, Butler MI, Poulter RT: Cryptons: a group of tyrosinerecombinase-encoding DNA transposons from pathogenic fungi. Microbiology 2003, 149:3099-3109.

20. Broach JR, Hicks JB: Replication and recombination functions associated with the yeast plasmid, $2 \mu$ circle. Cell 1980, 21:501-508.

21. Doak TG, Witherspoon DJ, Jahn CL, Herrick G: Selection on the genes of Euplotes crassus Tec1 and Tec2 transposons: evolutionary appearance of a programmed frameshift in a Tec2 gene encoding a tyrosine family site-specific recombinase. Eukaryot Cell 2003, 2:95-102.

22. Jacobs ME, Sánchez-Blanco A, Katz LA, Klobutcher LA: Tec3, a new developmentally eliminated DNA element in Euplotes crassus. Eukaryot Cell 2003, 2:103-114.

23. Goodwin TJ, Poulter RT: A new group of tyrosine recombinase-encoding retrotransposons. Mol Biol Evol 2004, 21:746-759.

24. Goodwin TJ, Poulter RT: The DIRS1 group of retrotransposons. Mol Biol Evol 2001, 18:2067-2082.

25. Lorenzi HA, Robledo G, Levin MJ: The VIPER elements of trypanosomes constitute a novel group of tyrosine recombinase-enconding retrotransposons. Mol Biochem Parasitol 2006, 145:184-194.

26. Golic KG, Lindquist S: The FLP recombinase of yeast catalyzes site-specific recombination in the Drosophila genome. Cell 1989, 59:499-509.

27. van Duyne GD: A structural view of tyrosine recombinase site-speciic recombination. In Mobile DNA II. Edited by: Craig NL, Craigie R, Gellert M, Lambowitz AM. Washington, DC: American Society of Microbiology Press; 2002:93-117.

28. Jurka J, Kapitonov W: First Cryptons from invertebrates. Repbase Rep 2008, 8:232-233.

29. Jurka J: First Cryptons from insects. Repbase Rep 2009, 9:468-480.

30. Hedges SB, Kumar S: The TimeTree of Life New York: Oxford University Press; 2009.

31. Raffa GD, Cenci G, Siriaco G, Goldberg ML, Gatti M: The putative Drosophila transcription factor Woc is required to prevent telomeric fusions. Mol Cell 2005, 20:821-831

32. Font-Burgada J, Rossell D, Auer H, Azorín F: Drosophila HP1c isoform interacts with the zinc-finger proteins WOC and Relative-of-WOC to regulate gene expression. Genes Dev 2008, 22:3007-3023.

33. Makino T, McLysaght A: Ohnologs in the human genome are dosage balanced and frequently associated with disease. Proc Natl Acad Sci USA 2010, 107:9270-9274

34. Kuraku S, Meyer A, Kuratani S: Timing of genome duplications relative to the origin of the vertebrates: did cyclostomes diverge before or after? Mol Biol Evol 2009, 26:47-59.

35. Ohno S: Evolution by Gene Duplication New York: Springer-Verlag; 1970.

36. Dlakić M, Mushegian A: Prp8, the pivotal protein of the spliceosomal catalytic center, evolved from a retroelement-encoded reverse transcriptase. RNA 2011, 17:799-808.

37. Pancer Z, Amemiya CT, Ehrhardt GR, Ceitlin J, Gartland GL, Cooper MD: Somatic diversification of variable lymphocyte receptors in the agnathan sea lamprey. Nature 2004, 430:174-180.

38. Kapitonov W, Jurka J: Harbinger transposons and an ancient HARBI1 gene derived from a transposase. DNA Cell Biol 2004, 23:311-324.

39. Sarkar A, Sim C, Hong YS, Hogan JR, Fraser MJ, Robertson HM, Collins FH: Molecular evolutionary analysis of the widespread piggyBac transposon family and related "domesticated" sequences. Mol Genet Genomics 2003, 270:173-180.

40. Casola C, Hucks D, Feschotte C: Convergent domestication of pogo-like transposases into centromere-binding proteins in fission yeast and mammals. Mol Biol Evol 2008, 25:29-41.

41. Hammer SE, Strehl S, Hagemann S: Homologs of Drosophila P transposons were mobile in zebrafish but have been domesticated in a common ancestor of chicken and human. Mol Biol Evol 2005, 22:833-844.

42. Guo F, Gopaul DN, van Duyne GD: Structure of Cre recombinase complexed with DNA in a site-specific recombination synapse. Nature 1997, 389:40-46.
43. Holland MJ, Yokoi T, Holland JP, Myambo K, Innis MA: The GCR1 gene encodes a positive transcriptional regulator of the enolase and glyceraldehyde-3-phosphate dehydrogenase gene families in Saccharomyces cerevisiae. Mol Cell Biol 1987, 7:813-820.

44. Rep M, Reiser V, Gartner U, Thevelein JM, Hohmann S, Ammerer G, Ruis H: Osmotic stress-induced gene expression in Saccharomyces cerevisiae requires Msn1p and the novel nuclear factor Hot1p. Mol Cell Biol 1999, 19:5474-5485.

45. Liu L, Ishihara K, Ichimura T, Fujita N, Hino S, Tomita S, Watanabe S, Saitoh N, Ito T, Nakao M: MCAF1/AM is involved in Sp1-mediated maintenance of cancer-associated telomerase activity. J Biol Chem 2009, 284:5165-5174

46. Ding X, Luo C, Zhou J, Zhong Y, Hu X, Zhou F, Ren K, Gan L, He A, Zhu J, Gao X, Zhang J: The interaction of KCTD1 with transcription factor AP-2a inhibits its transactivation. J Cell Biochem 2009, 106:285-295.

47. Hakimi MA, Dong Y, Lane WS, Speicher DW, Shiekhattar R: A candidate Xlinked mental retardation gene is a component of a new family of histone deacetylase-containing complexes. J Biol Chem 2003, 278:7234-7239.

48. Gocke CB, Yu H: ZNF198 stabilizes the LSD1-CoREST-HDAC1 complex on chromatin through its MYM-type zinc fingers. PLoS One 2008, 3:e3255.

49. Jiang W, Lechner J, Carbon J: Isolation and characterization of a gene (CBF2) specifying a protein component of the budding yeast kinetochore. J Cell Biol 1993, 121:513-519.

50. Goh PY, Kilmartin JV: NDC10: a gene involved in chromosome segregation in Saccharomyces cerevisiae. J Cell Biol 1993, 121:503-512.

51. Gillette WK, Martin RG, Rosner JL: Probing the Escherichia coli transcriptional activator MarA using alanine-scanning mutagenesis: residues important for DNA binding and activation. J Mol Biol 2000, 299:1245-1255

52. Martin RG, Gillette WK, Martin NI, Rosner JL: Complex formation between activator and RNA polymerase as the basis for transcriptional activation by MarA and SoxS in Escherichia coli. Mol Microbiol 2002, 43:355-370.

53. de Crozé N, Maczkowiak F, Monsoro-Burq AH: Reiterative AP2a activity controls sequential steps in the neural crest gene regulatory network. Proc Natl Acad Sci USA 2011, 108:155-160.

54. Dutta $S$, Dawid IB: Kctd15 inhibits neural crest formation by attenuating Wnt/ß-catenin signaling output. Development 2010, 137:3013-3018.

55. Xiao S, Nalabolu SR, Aster JC, Ma J, Abruzzo L, Jaffe ES, Stone R, Weissman SM, Hudson TJ, Fletcher JA: FGFR1 is fused with a novel zincfinger gene, ZNF198, in the $t(8 ; 13)$ leukaemia/lymphoma syndrome. Nat Genet 1998, 18:84-87.

56. van der Maarel SM, Scholten IHJM, Huber I, Philippe C, Suijkerbuijk RF, Gilgenkrantz S, Kere J, Cremers FPM, Ropers HH: Cloning and characterization of DXS6673E, a candidate gene for X-linked mental retardation in Xq13.1. Hum Mol Genet 1996, 5:887-897.

57. Warner DR, Roberts EA, Greene RM, Pisano MM: Identification of novel Smad binding proteins. Biochem Biophys Res Commun 2003, 312:1185-1190.

58. Kunapuli P, Somerville R, Still IH, Cowell JK: ZNF198 protein, involved in rearrangement in myeloproliferative disease, forms complexes with the DNA repair-associated HHR6A/6B and RAD18 proteins. Oncogene 2003, 22:3417-3423.

59. Kasyapa CS, Kunapuli $P$, Cowell JK: Mass spectroscopy identifies the splicing-associated proteins, PSF, hnRNP H3, hnRNP A2/B1, and TLS/FUS as interacting partners of the ZNF198 protein associated with rearrangement in myeloproliferative disease. Exp Cell Res 2005, 309:78-85.

60. Shav-Tal Y, Zipori D: PSF and p54 nrb/NonO: multi-functional nuclear proteins. FEBS Lett 2002, 531:109-114

61. Rajesh C, Baker DK, Pierce AJ, Pittman DL: The splicing-factor related protein SFPQ/PSF interacts with RAD51D and is necessary for homologydirected repair and sister chromatid cohesion. Nucleic Acids Res 2011, 39:132-145.

62. Dong L, Zhang X, Fu X, Zhang X, Gao X, Zhu M, Wang X, Yang Z, Jensen ON, Saarikettu J, Yao Z, Silvennoinen O, Yang J: PTB-associated splicing factor (PSF) functions as a repressor of STAT6-mediated lge gene transcription by recruitment of HDAC1. J Biol Chem 2011, 286:3451-3459.

63. Richards TA, Dacks JB, Jenkinson JM, Thornton CR, Talbot NJ: Evolution of filamentous plant pathogens: gene exchange across eukaryotic kingdoms. Curr Biol 2006, 16:1857-1864. 
64. Altschul SF, Madden TL, Schäffer AA, Zhang J, Zhang Z, Miller W, Lipman DJ: Gapped BLAST and PSI-BLAST: a new generation of protein database search programs. Nucleic Acids Res 1997, 25:3389-3402.

65. Kohany O, Gentles AJ, Hankus L, Jurka J: Annotation, submission and screening of repetitive elements in Repbase: RepbaseSubmitter and Censor. BMC Bioinformatics 2006, 7:474.

66. Katoh K, Kuma K, Toh H, Miyata T: MAFFT version 5: improvement in accuracy of multiple sequence alignment. Nucleic Acids Res 2005, 33:511-518.

67. Edgar RC: MUSCLE: multiple sequence alignment with high accuracy and high throughput. Nucleic Acids Res 2004, 32:1792-1797.

68. Guindon S, Dufayard JF, Lefort V, Anisimova M, Hordijk W, Gascuel O: New algorithms and methods to estimate maximum-likelihood phylogenies: assessing the performance of PhyML 3.0. Syst Biol 2010, 59:307-321.

69. Guindon S, Lethiec F, Duroux P, Gascuel O: PHYML Online: a web server for fast maximum likelihood-based phylogenetic inference. Nucleic Acids Res 2005, , 33 Web Server: W557-W559.

70. Anisimova M, Gascuel O: Approximate likelihood-ratio test for branches: a fast, accurate, and powerful alternative. Syst Biol 2006, 55:539-552.

doi:10.1186/1759-8753-2-12

Cite this article as: Kojima and Jurka: Crypton transposons: identification of new diverse families and ancient domestication events. Mobile DNA 2011 2:12.

\section{Submit your next manuscript to BioMed Central} and take full advantage of:

- Convenient online submission

- Thorough peer review

- No space constraints or color figure charges

- Immediate publication on acceptance

- Inclusion in PubMed, CAS, Scopus and Google Scholar

- Research which is freely available for redistribution

Submit your manuscript at www.biomedcentral.com/submit 